\title{
Avaliação da satisfação dos usuários de fisioterapia em atendimento ambulatorial
}

\author{
Evaluation of the satisfaction of physical therapy patients in outpatient care \\ Evaluación de la satisfacción de pacientes de fisioterapia en atención ambulatoria \\ Bruno Gonçalves Dias Moreno', José Eduardo Corrente², Marcia Galan Perroca³, Ivan Luiz Pavanelli4, \\ Paulo Roberto Rocha Júnior ${ }^{5}$
}

\begin{abstract}
RESUMO I A satisfação é determinada pela reação do paciente ao serviço recebido, sendo, portanto, um indicador sensível da qualidade do atendimento. 0 objetivo deste estudo foi comparar a satisfação dos usuários que realizam tratamento fisioterapêutico ambulatorial em clínicas públicas (CP), em clínicas privadas de convênio (CC) e em clínica-escola (CE). Foram avaliados 382 pacientes, com idade mínima de 18 anos, que haviam sido submetidos a, no mínimo, cinco atendimentos. Os pacientes foram divididos em três grupos e utilizou-se um questionário com perguntas sobre dados sociodemográficos e satisfação nos domínios interação paciente-terapeuta, acesso e atendimento da recepção, conveniência, ambiente e satisfação geral. A maior parte dos pacientes eram do sexo feminino (68,60\%), com média de 51,96 anos de idade. Na comparação entre os serviços, a CE apresentou maior satisfação que a CP em equipe de apoio, conveniência e ambiente físico, e a CC em relação terapeuta-paciente e satisfação geral. A CC foi mais bem avaliada que a CP em conveniência e ambiente físico. A análise de correlação entre a satisfação geral e cada um dos domínios mostrou valores de bom a moderado para relação terapeuta-paciente, e os menores valores para o domínio conveniência. O questionário mostrou boa consistência interna e coerência nos três serviços $(\alpha \geq 0,94)$. Esses resultados representam um importante indicador da impressão dos usuários nos serviços investigados, permitindo melhor direcionamento na implementação
\end{abstract}

de políticas públicas, privadas e acadêmicas, visando a melhora da qualidade dos atendimentos de fisioterapia. Descritores | Satisfação do Paciente; Fisioterapia; Inquéritos e Questionários; Setor Público; Setor Privado.

\begin{abstract}
I Satisfaction is determined by the patient's reaction to the service received, acting as a sensitive indicator of quality in medical care. The goal of this study was to compare the satisfaction of patients receiving outpatient physical therapy treatment in public clinics (PC), private healthcare clinics (PHC) and school clinics (SC). A total of 382 patients aged over 18 years old who had been to at least five appointments were divided into three groups. A questionnaire containing questions about sociodemographic data and overall satisfaction as well as satisfaction with the patienttherapist relationship, access to and support offered by the team, convenience and the environment was used. The majority of the patients was female (68.6\%), their mean age being 52.0 years old. In the comparison of the services, the school clinic showed greater satisfaction rates than the public clinics in relation to the support team, convenience and physical environment; and greater satisfaction rates than the private healthcare clinics in relation the therapistpatient relationship and overall satisfaction. The private healthcare clinics were better evaluated than the public clinics in relation to convenience and physical environment. The correlation analysis between overall satisfaction and each variable showed good and moderate values for the therapist-patient relationship variable and the lowest values
\end{abstract}

'Escola Brasileira de Fisioterapia Manipulativa (Ebrafim) - Araçatuba (SP), Brasil. E-mail: bruno@ebrafim.com. Orcid: 0000-0002-7427-5651 2Universidade Estadual Paulista (Unesp) - Botucatu (SP), Brasil. E-mail: jecorren@gmail.com. Orcid: 0000-0003-2308-1803 ${ }^{3}$ Faculdade de Medicina de São José do Rio Preto (Famerp) - São José do Rio Preto (SP), Brasil. E-mail: marcia.perroca@gmail.com. Orcid: 0000-0003-2931-8429

${ }^{4}$ Centro Universitário de Adamantina (Unifai) - Adamantina (SP), Brasil. E-mail: ivanluiz_p@hotmail.com. Orcid: 0000-0003-2738-289X ${ }^{5}$ Centro Universitário de Adamantina (Unifai) - Adamantina (SP), Brasil. E-mail: prochajr@terra.com.br. Orcid: 0000-0003-0434-6204 
for the convenience variable. The questionnaire had good internal consistency and coherence for the three services ( $\alpha \geq 0.94$ ). These results represent an important indicator of the patients' perception about the services investigated, allowing the proper implementation of public, private and academic policies aimed at the improvement of the quality of physical therapy care.

Keywords I Patient Satisfaction; Physiotherapy; Inquiries and Questionnaires; Public Sector; Private Sector.

RESUMEN | Determinada por la reacción al servicio recibido, la satisfacción del paciente es un indicador sensible de la calidad de la atención. El objetivo de este estudio fue comparar la satisfacción de los usuarios que realizan tratamiento fisioterapéutico ambulatorio en clínicas públicas (CP), en clínicas privadas de convenio (CC) y en clínica-escuela (CE). Se evaluaron 382 pacientes, con edad mínima de 18 años, sometidos a por lo menos cinco atendimientos. Los pacientes fueron divididos en tres grupos y se utilizó un cuestionario con preguntas sobre datos sociodemográficos y satisfacción en los dominios interacción paciente-terapeuta, acceso y atención de la recepción, conveniencia, ambiente y satisfacción general. La mayoría de los pacientes eran mujeres (68,60\%), con edad media de 51,96 años. En la comparación entre los servicios, la CE presentó mayor satisfacción que la CP en equipo de apoyo, conveniencia y ambiente físico, y la CC en la relación terapeuta-paciente y satisfacción general. La CC fue mejor evaluada que la CP en conveniencia y ambiente físico. El análisis de correlación entre la satisfacción general y cada uno de los dominios mostró valores de bueno a moderado para relación terapeuta-paciente, y los menores valores para el dominio conveniencia. El cuestionario mostró buena consistencia interna y coherencia en los tres servicios ( $\alpha \geq 0,94)$. Estos resultados representan un importante indicador de la impresión de los usuarios en los servicios investigados, permitiendo un mejor direccionamiento en la implementación de políticas públicas, privadas y académicas, buscando la mejora de la calidad de las atenciones de fisioterapia. Palablas clave | Satisfacción del Paciente; Fisioterapia; Encuestas y Cuestionarios; Sector Público; Sector Privado.

\section{INTRODUÇÃO}

O planejamento tem lugar de destaque na agenda da gestão dos sistemas de saúde em todas as esferas. Para que os gestores possam cumprir com sua responsabilidade de planejamento, são necessárias informações que os auxiliem na busca de soluções para as questões levantadas pela sociedade ${ }^{1}$.

Uma pesquisa de revisão de trabalhos publicados entre 1988 e 2014, sobre o tipo de produção científica em políticas de saúde no Brasil, destaca a participação da pesquisa como instrumento de gestão em saúde ${ }^{2}$.

Satisfação, na teoria sociopsicológica, é a expressão de uma atitude. $\mathrm{Na}$ saúde, é determinada pelas reações dos pacientes ao serviço recebido, podendo ser modificada quando as expectativas mudam ou seus padrões comparativos são alterados, mesmo que o serviço permaneça constante ${ }^{3}$.

Com a mudança frequente dos serviços de saúde e as variações do nível de expectativa dos usuários, a avaliação e o acompanhamento dos atendimentos são fundamentais para mensurar e controlar a satisfação dos pacientes.

$\mathrm{Na}$ literatura mundial, há questionários disponíveis para avaliar a satisfação com assistência recebida em seguradoras de saúde e outros recursos médicos, porém, tais instrumentos não são adequados para medir a satisfação do paciente com a fisioterapia, por se tratar de um atendimento com características diferentes envolvendo contato físico e a participação ativa do paciente ${ }^{4,5}$

O desenvolvimento e validação de um instrumento de pesquisa para avaliar a satisfação do paciente com a fisioterapia representa uma importante área de investigação em saúde, entretanto os instrumentos devem ser testados em diferentes situações, centros de pesquisa e em populações variadas. Pesquisadores brasileiros realizaram a tradução, adequação cultural e validação de questionário que avaliou 11 domínios. Após a pesquisa, a versão final passou a ter 23 questões para avaliar a interação terapeuta-paciente, acesso e atendimento da equipe, conveniência, ambiente físico e satisfação geral ${ }^{6}$.

Mais de $90 \%$ da população brasileira é usuária, de alguma forma, do Sistema Único de Saúde (SUS), segundo pesquisa realizada em 2003 pelo Ministério da Saúde ${ }^{7}$. Os autores descrevem uma baixa qualidade dos serviços oferecidos e a falta de mecanismos de acompanhamento, controle e avaliação. Nesse contexto, avaliar a satisfação com a assistência é primordial para melhorar os trabalhos prestados ${ }^{8}$.

O sistema privado de saúde e seus planos de assistência, desde o início, estão relacionados ao crescimento das cidades, à industrialização do país, ao emprego formal e à renda ${ }^{9}$. A fisioterapia brasileira no setor privado é representada por $60 \%$ de fisioterapeutas cadastrados ${ }^{10}$.

O notório crescimento do sistema de saúde privado requer cada vez mais atenção por parte dos consumidores e 
das agências reguladoras. Diante disso, passar a compreender melhor a satisfação desses pacientes parece importante fonte para normatização e crescimento desse setor.

Outra alternativa de assistência em fisioterapia que vem crescendo muito nos últimos anos são os atendimentos realizados por acadêmicos de instituições de ensino superior ${ }^{11}$. A maior parte dos atendimentos realizados por esse novo modelo de assistência acontece nas clínicas escola (CE) dos cursos de fisioterapia.

Nesse tipo de serviço o modelo de gestão é diferente dos citados anteriormente, pois tem como principal fonte de renda as mensalidades dos alunos ou os repasses realizados pelo governo estadual ou federal, portanto deve conciliar os cuidados necessários à atenção dos usuários, com as necessidades pedagógicas e diretrizes curriculares dos cursos de graduação.

O objetivo deste estudo foi comparar a satisfação dos usuários que realizam tratamento fisioterapêutico ambulatorial em clínicas públicas $(\mathrm{CP})$, clínicas privadas de atendimento de convênio (CC) e clínica-escola.

\section{METODOLOGIA}

Trata-se de um estudo analítico, observacional, transversal, com uma amostra representativa dos usuários do serviço de fisioterapia de cinco municípios vizinhos da região noroeste do estado de São Paulo. No total, foram avaliadas cinco clínicas de atendimento de convênios, cinco clínicas que realizam atendimentos públicos e uma clínica-escola.

Considerando a prevalência de satisfação com os serviços de fisioterapia de 50\% (prevalência desconhecida), uma confiabilidade de $95 \%$ e margem de erro de $5 \%$, o tamanho amostral mínimo deveria ser de 384 indivíduos, divididos em três grupos: pacientes atendidos em $\mathrm{CE}$, $\mathrm{CP}$ e CC.

Participaram da pesquisa pacientes de ambos os sexos, com idade mínima de 18 anos, que estivessem em tratamento de fisioterapia ambulatorial. Foram abordados, no total, 390 pacientes que realizaram no mínimo cinco sessões e tinham condições de ler e compreender o questionário de avaliação de satisfação. Aplicou-se o questionário Miniexame do Estado Mental (MEEM) para avaliar a função cognitiva dos sujeitos de acordo com seu nível de escolaridade ${ }^{12}$. Para ser aceito para pesquisa, o participante deveria conseguir pontuações iguais ou superiores às mínimas exigidas conforme sua classificação de instrução escolar.
Este projeto de pesquisa foi aprovado pelo CEP via plataforma Brasil e todos os sujeitos assinaram o Termo de Consentimento Livre e Esclarecido (TCLE).

Para avaliar a satisfação desses usuários com o serviço de fisioterapia foi aplicado um questionário ${ }^{6}$ validado em língua portuguesa, com 12 questões descritivas sociodemográficas, que caracterizam a amostra, e 23 questões (Q) sobre a satisfação dos usuários com os serviços de fisioterapia recebido, divididos nos domínios interação terapeuta-paciente, acesso e atendimento da equipe, conveniência, ambiente físico e satisfação geral.

Na coleta de dados, os pacientes foram abordados na sala de espera de cada um dos locais estudados imediatamente após seu horário de atendimento, entre os meses de setembro de 2014 e agosto de 2015. Posteriormente, foram explicados os objetivos da pesquisa e passadas as orientações para o preenchimento dos questionários. Todas as fichas de avaliação foram entregues e recolhidas em um envelope fechado e não identificado.

Para análise estatística, as respostas foram pontuadas em uma escala de 1 a 5 , incluindo os dados sociodemográficos que foram tabulados em ordem crescente, conforme a ordem de apresentação no questionário. A pontuação mínima e máxima do questionário passa a ser respectivamente: 23 e 115 para o total e, para cada domínio, os valores variam de acordo com o número de questões. Quanto maior a pontuação melhor é a satisfação do paciente.

Os dados sociodemográficos foram analisados por medidas descritivas de média e desvio-padrão (variáveis quantitativas) e frequência relativa (variáveis qualitativas).

Para comparar a satisfação entre CE, CC e CP, foi realizada uma análise da variância seguida pelo teste de Tukey, por se tratar de uma variável contínua. Posteriormente, foi utilizada a correlação de Spearman para verificar o impacto de cada um dos quatro domínios na satisfação geral, em cada um dos serviços avaliados. Foram considerados valores de $r \geq 0,5$ para correlações moderadas e 0,7 para ótimas correlações.

Para avaliação de consistência interna do questionário, calculou-se o coeficiente $\alpha$ de Cronbach e considerados satisfatórios valores de $\alpha \geq 0,7$. Em todos os testes foi utilizado o nível de significância de $5 \%$ ou o valor de $\mathrm{P}$ correspondente.

\section{RESULTADOS}

Entre os 390 pacientes abordados, oito não quiseram participar da pesquisa e nenhum dos entrevistados foi reprovado pelo MEEM, dessa forma, foram entrevistados 
382 pacientes, sendo 126 pacientes na CE, 126 nas CC, e 130 nas CP.

A média geral de idade nos três serviços foi de $51,93 \pm 13,66$ anos e notou-se que pacientes atendidos nas $\mathrm{CC}$ têm maior renda e escolaridade que os dos demais serviços. Nos três grupos, $\mathrm{CE}, \mathrm{CC}$ e $\mathrm{CP}$, a maior parte dos pacientes já havia feito tratamento de fisioterapia anteriormente e conheceu o serviço por intermédio de seu médico, conforme Tabela 1 .

O coeficiente $\alpha$ de Cronbach apresentou valores maiores de 0,7 na avaliação do total de pacientes em todos os setores. $\mathrm{Na}$ avaliação por domínio, os itens conveniência e satisfação geral tiveram valores um pouco menores que 0,7 na $\mathrm{CP}$ e em conveniência na $\mathrm{CE}$.

Por meio das médias de cada domínio, foram comparados os níveis de satisfação dos pacientes nos serviços avaliados, conforme Tabela 2. Observaram-se diferenças estatisticamente significantes da $\mathrm{CE}$, em relação à $\mathrm{CP}$ em alguns domínios, e em relação à $\mathrm{CC}$ em outros. A CP apresentou os menores valores de média de satisfação no geral quando comparados os três serviços.

Tabela 1. Distribuição dos dados sociodemográficos dos pacientes de acordo com o serviço, 2016

\begin{tabular}{|c|c|c|c|c|c|c|}
\hline Variável & & CE & $\mathrm{CC}$ & $\mathrm{CP}$ & Total & p \\
\hline Idade M (DP) & & $50,02(12,67)^{b}$ & $56,91(16,41)^{a}$ & $48,96(9,91)^{b}$ & $51,96(13,7)$ & $<0,0001^{*}$ \\
\hline \multirow{2}{*}{ Sexo \% (n) } & Masculino & $28,57(36)$ & $32,54(41)$ & $33,08(43)$ & $31,40(120)$ & \multirow{2}{*}{0,69} \\
\hline & Feminino & $71,43(90)$ & $67,46(85)$ & $66,92(87)$ & $68,60(262)$ & \\
\hline \multirow{5}{*}{ Escolaridade \% (n) } & $1^{\circ}$ grau incompleto & $30,16(38)$ & 26,19 (33) & $50,77(66)$ & $35,71(137)$ & \multirow{5}{*}{$<0,0001^{*}$} \\
\hline & $1^{\circ}$ grau completo & $14,28(18)$ & $19,04(24)$ & $18,45(24)$ & $17,26(66)$ & \\
\hline & $2^{\circ}$ grau incompleto & $12,70(16)$ & $3,97(5)$ & $14,62(19)$ & $10,43(40)$ & \\
\hline & $2^{\circ}$ grau completo & 26,19 (33) & $23,02(29)$ & $13,08(17)$ & $20,76(79)$ & \\
\hline & Superior & $16,67(21)$ & $27,78(35)$ & $3,08(4)$ & $15,84(60)$ & \\
\hline \multirow{4}{*}{$\begin{array}{l}\text { Renda familiar em salários } \\
\text { mínimos \% (n) }\end{array}$} & 1 a 3 & $72,22(91)$ & $64,29(81)$ & $82,31(107)$ & $72,94(279)$ & \multirow{4}{*}{$0,025^{*}$} \\
\hline & 4 a 6 & $19,85(25)$ & $22,22(28)$ & 14,61 (19) & $18,89(72)$ & \\
\hline & 7 a 10 & $7,14(9)$ & $10,32(13)$ & $2,31(3)$ & $6,59(25)$ & \\
\hline & Mais de 10 & $0,79(1)$ & $3,17(4)$ & $0,77(1)$ & $1,58(6)$ & \\
\hline \multirow{5}{*}{$\begin{array}{l}\text { Como conheceu esta clínica? } \\
\%(n)\end{array}$} & Médico & $53,17(67)$ & $38,10(48)$ & $63,85(83)$ & $51,71(198)$ & \multirow{5}{*}{$0,0005^{*}$} \\
\hline & Plano de saúde & $7,15(9)$ & $13,49(17)$ & $8,45(11)$ & $9,70(37)$ & \\
\hline & Amigo & $22,22(28)$ & $33,33(42)$ & $11,54(15)$ & $22,36(85)$ & \\
\hline & Paciente anterior & $11,90(15)$ & $7,14(9)$ & $11,54(15)$ & 10,19 (39) & \\
\hline & Outros & $5,56(7)$ & $7,94(10)$ & $4,62(6)$ & $6,04(23)$ & \\
\hline \multirow{2}{*}{$\begin{array}{l}\text { Foi a primeira experiência } \\
\text { com a fisioterapia? \% (n) }\end{array}$} & Sim & $38,10(48)$ & $37,30(47)$ & 33,85 (44) & 36,42 (139) & \multirow{2}{*}{0,753} \\
\hline & Não & $61,90(78)$ & $62.70(79)$ & $66,15(86)$ & $64,03(243)$ & \\
\hline \multirow{2}{*}{$\begin{array}{l}\text { Foi a primeira experiência } \\
\text { nesta clínica? \%(n) }\end{array}$} & Sim & $61,90(78)$ & $50,79(64)$ & $50,00(65)$ & $54,23(207)$ & \multirow{2}{*}{$0,010^{*}$} \\
\hline & Não & $38,10(48)$ & $49,21(62)$ & $50,00(65)$ & $45,77(175)$ & \\
\hline
\end{tabular}

*Valores estatisticamente significantes.

Tabela 2. Comparação dos escores de satisfação de atendimento entre a CE, CC e CP no geral e em cada domínio, 2016

\begin{tabular}{|c|c|c|c|c|}
\hline Variáveis & CE & $\mathrm{CC}$ & $\mathrm{CP}$ & p \\
\hline Total & $100,3^{b}$ & 97,4 & 95,0 & $0,0032^{*}$ \\
\hline 1. Interação terapeuta-paciente & $35,6^{\mathrm{a}}$ & 33,9 & 34,5 & $0,0153^{*}$ \\
\hline 2. Equipe de apoio & $25,7^{b}$ & 25,3 & 24,4 & $0,0142^{*}$ \\
\hline 3. Conveniência & $8,0^{b}$ & $8,3^{c}$ & 7,5 & $<0,0001^{*}$ \\
\hline 4. Ambiente Físico & $17,1^{\mathrm{b}}$ & $16,7^{c}$ & 15,2 & $<0,0001^{*}$ \\
\hline 5 - Satisfação Geral & $13,9^{\mathrm{a}}$ & 13,3 & 13,5 & $0,0052^{*}$ \\
\hline
\end{tabular}

a: valores estatisticamente significantes entre CE e CC; : valores estatisticamente significantes entre CE e CP; c: valores estatisticamente significantes entre CC e CP; *valores estatisticamente significantes pelo teste de comparação múltipla de Tukey. 
As análises de correlação entre os domínios interação terapeuta-paciente (1), equipe de apoio (2), conveniência (3) e ambiente físico (4), com as questões sobre satisfação geral estão destacadas na Tabela 3. Nota-se uma correlação moderada nos domínios de 1 a 4 com a questão 21 nos três serviços, entretanto, os maiores valores de $\mathrm{R}$ foram alcançados no domínio 1.O domínio 3 teve os menores valores de correlação com as questões sobre satisfação geral nos três grupos.

\begin{tabular}{|c|c|c|c|c|}
\hline & Domínios & Q21 (Satisfação Geral) & Q22 (Retornaria) & Q23 (Indicaria) \\
\hline \multirow{4}{*}{ CE } & 1. Terapeuta-paciente & $R=0,67 / p<0,0001^{*}$ & $R=0,47 / p<0,0001$ & $R=0,56 / p<0,0001^{*}$ \\
\hline & 2. Equipe de apoio & $R=0,57 / p<0,0001^{*}$ & $R=0,47 / p<0,0001$ & $R=0,54 / p<0,0001^{*}$ \\
\hline & 3. Conveniência & $R=0,52 / p<0,0001^{*}$ & $R=0,41 / p<0,0001$ & $R=0,55 / p<0,0001^{*}$ \\
\hline & 4. Ambiente físico & $\mathrm{R}=0,60 / p<0,0001^{*}$ & $\mathrm{R}=0,58 / p<0,0001^{*}$ & $R=0,58 / p<0,0001^{*}$ \\
\hline \multirow{4}{*}{ CC } & 1. Terapeuta-paciente & $\mathrm{R}=0,69 / \mathrm{p}<0,0001^{*}$ & $R=0,52 / p<0,0001^{*}$ & $R=0,50 / p<0,0001^{*}$ \\
\hline & 2. Equipe de apoio & $R=0,66 / p<0,0001^{*}$ & $R=0,45 / p<0,0001$ & $R=0,42 / p<0,0001$ \\
\hline & 3. Conveniência & $R=0,68 / p<0,0001^{*}$ & $R=0,36 / p<0,0001$ & $R=0,42 / p<0,0001$ \\
\hline & 4. Ambiente físico & $R=0,68 / p<0,0001^{*}$ & $R=0,42 / p<0,0001$ & $R=0,47 / p<0,0001$ \\
\hline \multirow{4}{*}{$\mathrm{CP}$} & 1. Terapeuta-paciente & $\mathrm{R}=0,78 / \mathrm{p}<0,0001^{*}$ & $R=0,43 / p<0,0001$ & $R=0,40 / p<0,0001$ \\
\hline & 2. Equipe de apoio & $R=0,66 / p<0,0001^{*}$ & $R=0,33 / p=0,0001$ & $R=0,39 / p<0,0001$ \\
\hline & 3. Conveniência & $R=0,45 / p<0,0001$ & $R=0,22 / p=0,0122$ & $R=0,25 / p=0,0041$ \\
\hline & 4. Ambiente físico & $R=0,57 / p<0,0001^{*}$ & $R=0,36 / p<0,001$ & $R=0,30 / p=0,0004$ \\
\hline
\end{tabular}

*Valores de $r \geq 0,5$ e $p<0,05$.

\section{DISCUSSÃO}

É consenso que a satisfação reflete a percepção do paciente sobre a qualidade do serviço recebido, no entanto, este tema é amplo e influenciado por valores socioculturais e condições ambientais dos serviços ${ }^{13}$.

Numa pesquisa ${ }^{14}$ com 1.944 pacientes para avaliar a satisfação com o tratamento recebido, constatou-se que os entrevistados eram mais suscetíveis a saírem satisfeitos com o cuidado independentemente da mudança clínica. Concluíram, que a satisfação do paciente com o atendimento independe dos resultados do tratamento. Tais discrepâncias justificam a discussão sobre a importância de avaliar a eficácia de um atendimento em todos os seus aspectos.

O conhecimento do perfil do paciente permite criar soluções adaptadas à realidade do local. Estudos apontam que o perfil socioeconômico de uma população exerce influência na saúde de forma independente das características individuais ${ }^{15}$.

A média de idade dos pacientes avaliados foi de aproximadamente 51,96 anos e os pacientes mais velhos eram da CC, com média de 56,91 anos e diferença estatística em relação às demais. A população idosa do Brasil vem crescendo, contribuindo para o aumento nos indicadores de doenças crônicas que podem exigir cuidados permanentes ${ }^{16}$. O grau de limitação física justifica o perfil de idade dos pacientes atendidos neste estudo e na maior parte dos trabalhos sobre satisfação em fisioterapia.

Nos três grupos avaliados, verificou-se uma maioria do sexo feminino. A prevalência de mulheres em atendimento fisioterapêutico é destacada na maior parte das publicações nesta área ${ }^{17-19}$. Autores atribuem este dado ao fato de muitas mulheres, além dos afazeres domésticos, realizarem suas atividades profissionais, favorecendo complicações osteomusculares ${ }^{17}$.

Os resultados sobre o estudo da confiabilidade e da validade do questionário utilizado indicaram propriedades psicométricas satisfatórias à sua utilização nesta população. A confiabilidade calculada, pelo coeficiente $\alpha$ de Cronbach ( $\alpha=0,94$ na CE, 0,97 na $\mathrm{CC}$ e 0,94 na $\mathrm{CP})$, excedeu os valores propostos como critério para estudos exploratórios ${ }^{20}$. Quando os domínios foram avaliados individualmente, apenas a conveniência $(\mathrm{CE}$ e $\mathrm{CP})$ e satisfação geral (CP) não atingiram escores satisfatórios, fato também observado em outro estudo da área ${ }^{6}$.

Em geral, podemos observar que todos os serviços avaliados apresentaram bons índices de satisfação, sendo que os maiores níveis de satisfação de atendimento em fisioterapia são da CE, e os piores, da CP.

Podemos observar que a $\mathrm{CE}$ teve valores superiores aos da CC em interação terapeuta-paciente e satisfação geral e em relação à $\mathrm{CP}$ nos domínios atendimento da equipe, 
ambiente, conveniência e no geral. A CC apresentou valores superiores aos da $\mathrm{CP}$ em ambiente e conveniência.

Considerando os altos níveis de satisfação observados nas $\mathrm{CE}$, podemos considerar que esse serviço poderia ser mais observado quanto ao seu sistema de gestão e ações, criando novas alternativas de administração aos outros serviços de fisioterapia ambulatorial.

Um estudo realizado em uma clínica-escola, na cidade de Santo André (SP), mostrou que os resultados das respostas sobre a satisfação dos usuários apontam, de forma positiva, para a competência do trabalho realizado por sua equipe ${ }^{18}$.

A CP teve as médias mais baixas de satisfação dos serviços avaliados, fato que também merece reflexão, pois a promoção da cidadania de parcela significativa dos usuários de saúde depende da eficiência do setor público.

Tão importante quanto o planejamento é a mensuração e a avaliação das ações empreendidas e dos resultados alcançados. As avaliações são importantes contribuições sob a responsabilidade das instituições de pesquisa, que interagem com segmentos da sociedade para o seu aperfeiçoamento contínuo ${ }^{21}$.

A análise da relação entre as questões sobre satisfação geral dos pacientes, com cada um dos domínios, apresentou bons níveis de correlação com a questão 21 nos três grupos. É importante destacar que o domínio sobre a relação terapeuta-paciente teve os valores mais altos de correlação.

Vários autores apontam esse domínio como um dos mais influentes sobre a satisfação do paciente , $^{4,6,22,23}$. A forma como o fisioterapeuta se porta durante a sessão influencia a satisfação geral mais que outros domínios do atendimento.

A CE teve as melhores correlações com os domínios avaliados nas três questões sobre satisfação geral. Nesse serviço, destaca-se a individualidade da assistência, pois cada aluno está disponível a um paciente por horário. Outro fator importante é que, para cada grupo de seis alunos, está presente um professor supervisor de estágio, por norma do Conselho Federal de Fisioterapia ${ }^{24}$ (Coffito) de 2013. Nos demais serviços de fisioterapia, admite-se que em horário de atendimento o profissional receba até seis pacientes, dependendo da natureza da patologia 25 .

Em uma pesquisa sobre acesso e acolhimento em unidades de saúde, o desempenho profissional durante o atendimento e o vínculo estabelecido entre o usuário e o serviço foram importantes fatores valorizados pelos usuários ${ }^{26}$. Os resultados também concordam com as análises deste estudo.

A carência de recursos financeiros por parte do poder público é considerada um dos desafios que dificultam a humanização do atendimento, pois tem impacto importante na estrutura física e material dos serviços ${ }^{27}$.

Neste estudo, o domínio conveniência teve os menores valores de correlação com a satisfação geral. Esses resultados são contraditórios entre publicações na área, pois corroboram com alguns autores e divergem de outras publicações ${ }^{8,19}$.

É importante destacar que os municípios avaliados são pequenos, com menos de 50 mil habitantes, o que pode favorecer o menor impacto dos itens avaliados.

A falta de padronização dos instrumentos de avaliação reduz a possibilidade de comparação dos resultados ${ }^{28}$. No Brasil, o primeiro questionário testado e validado psicometricamente em língua portuguesa foi desenvolvido em 2007 e, até o momento, cerca de dez artigos com o uso dessa ferramenta foram publicados.

A relação terapeuta-paciente pode dificultar a avaliação do usuário, segundo pesquisadores ${ }^{29}$, quando os usuários têm altos níveis de envolvimento com um serviço, tendem a atribuir valores positivos a ele. Esse tipo de viés é difícil de ser eliminado em estudos de terapia física devido à natureza dos serviços. Questionários padronizados não fornecem informações completas sobre o objeto de pesquisa, portanto, a inclusão de questões abertas poderia enriquecer a compreensão sobre as maiores necessidades do serviço avaliado ${ }^{4}$.

Apesar dos altos índices de satisfação deste estudo, não podemos considerar que as condutas terapêuticas foram as mais adequadas, pois o questionário avalia a satisfação do usuário com os cuidados prestados, e não os procedimentos e resultado alcançado. Outro ponto de atenção sobre este tema é que o indivíduo tende a ser mais crítico com o passar do tempo, se tornando mais exigente ${ }^{30}$. Portanto, o processo de avaliação das expectativas do usuário sobre os serviços de saúde merece atenção permanente.

\section{CONCLUSÃO}

Em relação aos resultados desta pesquisa foi possível verificar que, quando comparados os serviços de fisioterapia, pacientes atendidos na $\mathrm{CE}$ apresentaram maior satisfação que a $\mathrm{CP}$ em relação à equipe de apoio, conveniência e ambiente físico, e que a $\mathrm{CC}$ na relação terapeuta-paciente e satisfação geral. A CC foi mais bem avaliada que a $\mathrm{CP}$ em conveniência e ambiente físico. Entre todos os domínios investigados, a relação terapeuta-paciente teve maior correlação com a satisfação geral, e conveniência a menor. $\mathrm{Na} \mathrm{CP}$ foi observada boa correlação positiva com satisfação geral entre idade, sexo feminino e renda. 
Estes resultados representam um importante indicador da impressão dos usuários nos serviços investigados nesta pesquisa, descrevendo o perfil, valores e diferenças nas crenças de satisfação dos pacientes. Estes dados permitem um melhor direcionamento para possíveis implementações de políticas públicas, privadas e acadêmicas visando à melhora da qualidade dos atendimentos de fisioterapia.

\section{REFERÊNCIAS}

1. Pereira BS, Tomasi E. Instrumento de apoio à gestão regional de saúde para monitoramento de indicadores de saúde. Epidemiol Serv Saude. 2016;25(2):411-8. doi: 10.5123/ s1679-49742016000200019

2. Santos JS, Teixeira CF. Política de saúde no Brasil: produção científica 1988-2014. Saúde Debate. 2016;40(108):219-30. doi: 10.1590/0103-1104-20161080018

3. Sena ALC, Ferreira LN, Oliveira RS, Kozmhinsky VMR. Acolhimento e satisfação do usuário na estratégia de saúde da família: uma experiência de êxito. Rev APS. 2015;18(2):134-140.

4. Beattie PF, Pinto MB, Nelson MK, Nelson R. Patient satisfaction with outpatient physical therapy: Instrument validation. Phys Ther. 2002;82:557-65.

5. Monnin D, Perneger TV. Scale to measure patient satisfaction with physical therapy. Phys Ther. 2002;82:682-91.

6. Mendonça KMPP, Guerra RO. Desenvolvimento e validação de um instrumento de medida da satisfação do paciente com a fisioterapia. Rev Bras Fisioter. 2007;11(5):369-76.

7. Brasil. Ministério da Saúde. Ministério da Saúde lança QualiSUS em hospitais do Rio de Janeiro. Brasília: Ministério da Saúde; 2013 [cited 2013 Aug 10]. Available from: http://portal.saude. gov.br/saude

8. Moreira FM, Borba JAM, Mendonça KMPP. Instrumento para aferir satisfação do paciente com assistência fisioterapêtica na rede pública de saúde. Fisioter Pesqui. 2007;14(3):37-43.

9. Szwarcwald CL, Malta DC, Pereira CA, Vieira MLFP, Conde WL, Souza PRBJ, et al. Pesquisa Nacional de Saúde no Brasil: concepção e metodologia de aplicação. Ciên Saúde Coletiva. 2014;19(2):333-342.

10. Costa LR, Costa JLR, Oishi J, Driusso P. Distribuição de fisioterapeutas entre estabelecimentos públicos e privados nos diferentes níveis de complexidade de atenção à saúde. Rev Bras Fisioter. 2012;16(5):422-30.

11. Silva JFJ, Araujo SP, Nava AV, Dias RS. Serviço prestado em uma clínica escola: fatores influentes na satisfação dos pacientes. Rev Saúde Pesqui. 2014;7(1):129-134.

12. Bertolucci PH, Brucki SM, Campacci SR, Juliano Y. O miniexame do estado mental em uma população geral: impacto da escolaridade. Arq Neuropsiquiatr. 1994;52:1-7.

13. Borde E, Alvarez MH, Porto MFS. Uma análise crítica da abordagem dos Determinantes Sociais da Saúde a partir da medicina social e saúde coletiva latino-americana. Saúde Debate. 2015:39(106):841-54. doi: 10.1590/0103-1104201510600030023
14. Beattie PF, Nelson RM, Heintzelman M. The relationship between patient satisfaction with physical therapy care and global rating of change reported by patients receiving worker's compensation. Physiother Theory Pract. 2011;27(4):3108. doi: 10.3109/09593985.2010.490575

15. Fausto MCR, Giovanella L, Mendonça MHM, Seidl H, Gagno J. A posição da Estratégia Saúde da Família na rede de atenção à saúde na perspectiva das equipes e usuários participantes do PMAQ-AB. Saúde Debate. 2014;38(1):13-33. doi: 10.5935/0103-1104.2014S003

16. Instituto Brasileiro de Geografia e Estatística - IBGE. Brasília: IBGE; 2004 [cited 2013 Aug 10]. Available from: http://www. ibge.gov.br

17. Machado NP, Nogueira LT. Avaliação da satisfação dos usuários de serviços de Fisioterapia. Rev Bras Fisioter. 2008;12(5):401-8.

18. Suda EY, Uemura MD, Velasco E. Avaliação da satisfação dos pacientes atendidos em uma clínica-escola de Fisioterapia de Santo André, SP. Fisioter Pesqui. 2009;16(2):126-31.

19. Diogenes TPM, Mendonça KMPP, Guerra RO. Dimensions of satisfaction of older adult Brazilian outpatients with physical therapy. Rev Bras Fisioter. 2009;13(4):301-7.

20. Malhotra NK. Pesquisa de marketing: uma orientação aplicada. 3a ed. Porto Alegre: Bookman; 2001.

21. Coluci MZO, Alexandre NMC, Milani D. Construção de instrumentos de medida na área da saúde. Ciên Saúde Coletiva. 2015;20(3):925-36. doi: 10.1590/1413-81232015203.04332013

22. Oliveira NDFC, Costa LOP, Nelson R, Maher CG, Beattie PF, Bie R. Measurement properties of the Brazilian Portuguese version of the MedRisk Instrument for measuring patient satisfaction with physical therapy care. J Orthop Sports Phys Ther. 2014;44(11):879-89. doi: 10.2519/jospt.2014.5150

23. Carvalho VL, Cavalcante DM, Santos LPD, Pereira MD. Satisfação dos pacientes atendidos no estágio curricular de fisioterapia na comunidade. Fisioter Pesqui. 2013;20(4):330-335.

24. Conselho Federal de Fisioterapia e Terapia Ocupacional COFITO. Resolução n 431 , de 27 de setembro de 2013 [Internet]. Brasília: Coffito; 2013 [cited 2016 Jan 10]. Available from: http:// coffito.gov.br/nsite/?p=3193

25. Brasil. Ministério da Saúde. Sistema Único de Saúde. Legislação Federal. Conselho Federal de Fisioterapia e Terapia Ocupacional. Ofício Coffito Grade nº 007/96. São Paulo; 1996 [cited 2016 Set 14]. Available from: http://sna.saude.gov.br/legisla/legisla/ control_av/COFFITO_OF.007_96control_av_g.doc

26. Coutinho LRP, Barbieri AR, Santos MLM. Acolhimento na atenção primária à saúde: revisão integrativa. Saúde Debate. 2015;39(105):514-24. doi: 10.1590/0103-110420151050002018

27. Santos AM, Giovanella L. Governança regional: estratégias e disputas para gestão em saúde. Rev Saúde Pública. 2014;48(4):622-31. doi: 10.1590/S0034-8910.2014048005045

28. Cardoso LHP, Gosling M, Andrade MAM. Satisfação do usuário no âmbito dos serviços de saúde: as contribuições da literatura de marketing. RAHIS. 2013;10(3):75-92. doi: 10.21450/rahis.v10i3.2130

29. Aleluia IRS, Santos FC. Auditoria em fisioterapia no Sistema Único de Saúde: proposta de um protocolo específico. Fisioter Mov. 2013;26(4):725-41.

30. Jackson JL, Chamberlin J, Kroenke K. Predictors of patient satisfaction. Soc Sci Med. 2001;52:609-20. 